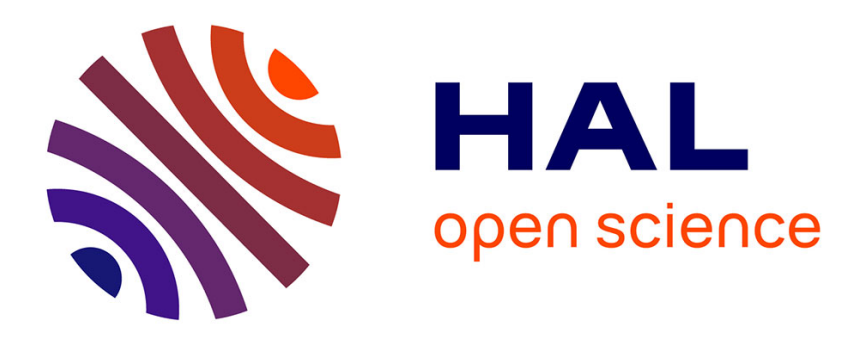

\title{
Raison publique rawlsienne et démocratie délibérative. Charles Girard
}

\section{To cite this version:}

Charles Girard. Raison publique rawlsienne et démocratie délibérative.. Raisons politiques, 2009, 34 (2), pp.259 - 306. 10.3917/rai.034.0073 . hal-01758514

\section{HAL Id: hal-01758514 https://hal.science/hal-01758514}

Submitted on 14 Nov 2020

HAL is a multi-disciplinary open access archive for the deposit and dissemination of scientific research documents, whether they are published or not. The documents may come from teaching and research institutions in France or abroad, or from public or private research centers.
L'archive ouverte pluridisciplinaire HAL, est destinée au dépôt et à la diffusion de documents scientifiques de niveau recherche, publiés ou non, émanant des établissements d'enseignement et de recherche français ou étrangers, des laboratoires publics ou privés. 


\title{
Raison publique rawlsienne et démocratie délibérative. Deux conceptions inconciliables de la légitimité politique?
}

\author{
Charles Girard, Université Paris 1 Panthéon-Sorbonne
}

(Version auteur)

Raisons politiques, 2009/02, n³4, p. 73-99.

Le problème de la légitimité politique divise les théories normatives de la démocratie. Comment concilier la nécessité de prendre des décisions collectives contraignantes pour tous et le statut de citoyen libre et égal aux autres de chaque individu ? Longtemps dominantes, les théories qui fondent la légitimité de la décision collective sur l'agrégation des préférences individuelles, sont aujourd’hui contestées par les tenants de la démocratie délibérative. Selon ces derniers, une décision est légitime dans la mesure où elle résulte d'un échange public, libre et raisonné d'arguments entre citoyens égaux. C'est en donnant au processus de prise de décision collective la forme d'une délibération publique portant sur le bien commun qu'il serait possible d'assurer à la fois l'efficacité et la légitimité de l'exercice démocratique du pouvoir.

Les théories élaborant cet idéal normatif se réfèrent communément à John Rawls et à Jürgen Habermas comme aux «pères fondateurs » de la démocratie délibérative. Comme l'éthique de la discussion habermassienne, la philosophie politique rawlsienne aurait, dès la Théorie de la justice, ouvert la voie à une compréhension délibérative de la politique démocratique ; et l'idée de raison publique, développée à partir de Libéralisme politique ${ }^{1}$ est souvent citée comme l’une des versions les plus abouties de l’idéal délibératif.

L’association de la démocratie délibérative et de la raison publique paraît aller de soi. La raison publique exige de chaque citoyen qu'il n'avance, pour justifier publiquement les décisions politiques qu'il soutient, que des raisons dont il peut raisonnablement penser qu'elles sont acceptables par les autres citoyens raisonnables. Elle peut donc aisément être comprise comme le principe qui doit guider la délibération publique, et celle-ci comme la forme que prend l'exercice collectif de la raison publique. Les commentateurs hésitent

\footnotetext{
${ }^{1}$ John Rawls, «The Idea of Public Reason », in Political Liberalism, New York, Columbia University Press, 1993, p. 212-254. Trad.par C. Audard : John Rawls, « La raison publique », in Libéralisme politique, Paris, PUF, (1995) 2001, p. 259-306.
} 
d'autant moins à associer, sinon à confondre, ces deux notions, que Rawls lui-même affirme que l'idée de raison publique est propre à une démocratie constitutionnelle bien ordonnée « comprise également comme une démocratie délibérative ». Parce que la théorie délibérative « limite les raisons que les citoyens peuvent donner à l'appui de leurs opinions politiques aux raisons compatibles avec une vision des autres citoyens comme égaux », elle inclut l'idée de la raison publique comme l'un de ses « éléments essentiels $»^{2}$.

C’est l'évidence de cette association que nous voulons remettre en cause ici, en montrant que ces deux concepts renvoient en réalité à deux conceptions distinctes et inconciliables de la légitimité démocratique. Tandis que la théorie délibérative fait dériver la légitimité d'un processus d'argumentation libre et publique, qui doit, en transformant les volontés individuelles, permettre de s’approcher d'un accord moral et épistémique sur la décision à prendre, la raison publique fonde la légitimité sur un principe qui exclut du forum public toutes les raisons ne s’appuyant pas sur la conception politique de la justice supposée commune à tous les citoyens. La première conçoit d'abord la démocratie comme un idéal d’autogouvernement par le débat public et refuse donc de contraindre la délibération par des normes définissant de manière indépendante quels types de raisons ou de décisions sont acceptables. La seconde, au contraire, conçoit d'abord la démocratie comme un idéal de coopération équitable entre égaux, et subordonne donc la délibération politique à un consensus préalable sur des normes d'acceptabilité qui dérivent de cet idéal, indépendamment de la procédure délibérative.

Nous montrerons comment la démocratie délibérative, prolongeant et critiquant tout à la fois la politique démocratique de la Théorie de la justice (1), propose de résoudre le problème de la légitimité démocratique en faisant reposer celle-ci sur la transformation des volontés individuelles plutôt que sur leur seule agrégation (2). Mais le critère de légitimité auquel cette conception aboutit - le consensus raisonné, réel ou hypothétique, entre citoyens - est insuffisant pour évaluer les résultats de délibérations concrètes (3). L’idée de la raison publique, élaborée par Rawls à partir de Libéralisme politique, échappe en partie à cette difficulté (4), mais seulement en sacrifiant l'ambition - centrale dans les théories délibératives - de ne pas subordonner le débat public à des principes indépendants (5). Raison publique et démocratique délibérative renvoient donc à deux conceptions inconciliables de la légitimité politique, qui ne peuvent être conciliées au sein d’une même théorie démocratique (6).

\footnotetext{
2 John Rawls, «The Idea of Public Reason Revisited », in The Law of Peoples, Cambridge, Mass., Harvard University Press, (1999) 2002. Trad. par B. Guillarme : John Rawls, "L’idée de raison publique reconsidérée », in Paix et démocratie, Paris, La découverte, 2006, p. 168.
} 


\section{La délibération dans la Théorie de la justice}

Les théories délibératives s'élaborent à la fois dans le prolongement de la politique démocratique esquissée par la Théorie de la justice et en rupture avec elle. Certes, l'œuvre de Rawls se consacre en premier lieu au problème de la justice sociale, et non à celui de la légitimité du pouvoir politique, et le dispositif conceptuel qu'elle met en œuvre ne fait guère intervenir l’idée de délibération. La position originelle n’exige en particulier des individus qui s’y trouvent ni qu'ils raisonnent en termes de bien commun ni qu’ils débattent véritablement entre eux des options qui leur sont soumises. Au contraire, c'est le calcul intéressé d'un agent économique motivé par son intérêt bien compris qui fonde la sélection des deux principes de justice. Quoique Rawls utilise la métaphore de la discussion et mentionne la présence d'un arbitre, il précise que celui-ci n’est pas nécessaire, car les «délibérations » de tous les individus situés derrière le voile d'ignorance sont supposées identiques : ils font tous le même raisonnement. Le choix des principes de justice repose donc sur l'unanimité spontanée des volontés, et la position originelle est précisément définie de telle sorte à ce que celle-ci soit possible $^{3}$. Il n’y a pas de véritable délibération collective derrière le voile d'ignorance ${ }^{4}$.

C’est en rupture avec la démarche rawlsienne que Bernard Manin propose justement l'une des premières formulations de la théorie délibérative. En faisant reposer sa théorie de la justice comme équité sur l'unanimité des individus situés dans la position originelle, Rawls reproduit aux yeux de Manin le geste de Rousseau imaginant une assemblée de citoyens dont les volontés s’accorderaient pour exprimer la volonté générale sans qu’il leur soit nécessaire de communiquer entre eux. Or l'approche qui fait reposer la légitimité sur l'accord spontané des volontés soulève deux difficultés : d'une part, le manque de plausibilité d'une telle unanimité - même sous les conditions hypothétiques de la position originelle, il n’est pas certain que le principe de différence apparaisse à tous comme la solution la plus rationnelle et d'autre part, le caractère dynamique, et non statique, des volontés. « Ce qu'il faut reprocher à Rawls comme à Rousseau [...] c'est le présupposé que les individus entrant en société ou devant prendre une décision politique ont déjà une volonté formée, qu'ils savent exactement

\footnotetext{
3 John Rawls, John Rawls, A Theory of Justice, Cambridge, Mass., Harvard University Press, (1971) édition révisée 1999. Trad. par C. Audard : John Rawls, Théorie de la justice, Paris, Seuil, (1987) 1997, § 24.

${ }^{4}$ Cette lecture est contestée. Pour une interprétation de la position originelle comme moment délibératif, voir Catherine Audard, John Rawls, Stocksfield, Acumen, 2007, chap. 3.
} 
ce qu'ils veulent et n'ont, tout au plus, qu'à appliquer leur critère d'évaluation aux solutions proposées $»^{5}$.

Ce n’est pas sur l'adéquation entre le contenu des décisions et le contenu de la volonté de chaque citoyen qu'il faut faire reposer la légitimité, affirme Manin, mais plutôt sur la participation de tous au processus délibératif par lequel les volontés individuelles se forment. En délibérant, c'est-à-dire en s'exposant mutuellement les raisons qui soutiennent à leurs yeux les différentes options envisagées, les citoyens s'informent et se forgent une opinion plus réfléchie ; ils essaient également de se convaincre les uns les autres du bien fondé de leurs positions respectives. Ils exercent ainsi leur droit moral à être les auteurs de la décision politique, sans que l'unanimité, spontanée ou non, ne soit requise. « On doit donc récuser la conclusion fondamentale de Rousseau [...] ou de Rawls : la décision légitime n'est pas la volonté de tous, mais celle qui résulte de la délibération de tous ; c'est le processus de formation des volontés qui confère sa légitimité au résultat, non les volontés déjà formées » ${ }^{6}$.

Il y a pourtant bien une esquisse de politique délibérative dans la Théorie de la justice. Elle n'est pas à chercher dans la position originelle, mais dans la description de la politique adaptée à une société bien ordonnée ${ }^{7}$. Dans une telle société, le débat politique prend la forme d'une confrontation entre conceptions du bien commun : les citoyens et les associations intervenant dans l'arène publique doivent argumenter sur la base de telles conceptions, et non adopter des points de vue particuliers ${ }^{8}$. C’est pourquoi Joshua Cohen estime que la théorie délibérative prolonge certains traits de la politique rawlsienne : la délibération centrée sur le bien commun, mais également l'insistance sur l'égalité manifeste entre citoyens ainsi que sur le rôle que joue la culture politique dans le développement de conceptions du bien commun.

Cette filiation implique toutefois à nouveau une rupture, car la théorie délibérative ne peut se satisfaire de la manière dont Rawls justifie ces traits. Ils sont nécessaires à la fois pour garantir que les citoyens puissent exercer leur droit de participation politique - dont le principe serait choisi par les individus situés dans la position originelle - et pour assurer que les institutions politiques reflètent l'idéal d'équité - car si nous acceptons cet idéal, nous devons désirer que les institutions elles-mêmes s’y conforment. Les aspects délibératifs de la politique rawlsienne dérivent donc de l'idée d’un système équitable de coopération, point de départ de la théorie de la justice comme équité. Mais cette justification instrumentale est trop

\footnotetext{
${ }^{5}$ Bernard Manin, «Volonté générale ou délibération. Esquisse d’une théorie de la délibération politique », Le débat, vol. 33, 1985, p. 83.

${ }^{6}$ Ibid, p. 83.

${ }^{7}$ Sur la théorie de la démocratie de Rawls, voir Joshua Cohen, « For a Democratic Society », in S. Freeman (dir.), The Cambridge Companion to Rawls, Cambridge, Cambridge University Press, 2003, p. 86-138.

${ }^{8}$ John Rawls, Théorie de la justice, op. cit., p. 262, 401-402, 512.
} 
fragile aux yeux de Cohen, car elle implique que la politique délibérative n’est nécessaire que si elle est la seule à pouvoir satisfaire l'équité. Or il n'est pas certain qu'une politique fondée sur la négociation entre groupes poursuivant leurs intérêts particuliers soit moins à même de refléter l'idéal d'équité qu'une politique délibérative, car la négociation peut elle aussi suivre des règles équitables.

C’est l’idée même d'une justification instrumentale que refusent en réalité Cohen et les tenants de la démocratie délibérative. Ce qui justifie à leurs yeux les traits délibératifs de la politique démocratique, c'est qu'ils sont constitutifs d'un idéal à la fois «intuitif » et « fondamental »: celui «d'une association démocratique dans laquelle la justification des termes et des conditions d'association procède par le débat et le raisonnement public entre citoyens égaux ${ }^{9}$. Parce que cet idéal est fondamental, il ne peut être dérivé d’autres idéaux, telle l'équité. Il est premier, et dans une certaine mesure intuitif. Seyla Benhabib considère ainsi que l’idéal délibératif traduit la forme de rationalité pratique qui nous a été légué par notre histoire $^{10}$, et Maeve Cooke juge qu'il exprime «qui nous sommes » mieux que toute autre conception de la démocratie moderne ${ }^{11}$.

Ces théoriciens s'émancipent donc de la perspective rawlsienne qui leur a en partie ouvert la voie, en affirmant le caractère fondamental, pour notre culture politique, de l’idéal d’une communauté politique se gouvernant elle-même par la délibération publique ${ }^{12}$.

\section{La démocratie délibérative et le consensus sur la décision}

Afin de préciser le sens de cet idéal, il est utile de considérer la définition avancée par Cohen, à laquelle Habermas et Rawls renvoient comme à la formulation « canonique » de la théorie délibérative ${ }^{13}$. Une démocratie délibérative est, selon Cohen, une association dont les

\footnotetext{
${ }^{9}$ Cohen, Joshua Cohen, « Deliberation and Democratic Legitimacy », in A. Hamlin et P. Pettit (dir.), The Good Polity, Oxford, Blackwell, 1989, p. 21. Trad. par C. Girard : Joshua Cohen, «Délibération et légitimité démocratique ", in C. Girard et A. Le Goff (dir.), La démocratie délibérative. Textes fondamentaux, Paris, Hermann, 2009 (à paraître).

10 Seyla Benhabib, "Toward a Deliberative Model of Democratic Legitimacy», in S. Benhabib (dir.), Democracy and Difference. Contesting the Boundaries of the Political, Princeton, Princeton University Press, 1996.

${ }^{11}$ Maeve Cooke, « Five arguments for deliberative democracy », Political Studies, 2000. Trad. par A. Le Goff : Maeve Cooke, " Cinq arguments en faveur de la démocratie delibérative », in C. Girard et A. Le Goff, La démocratie délibérative. Textes fondamentaux, op. cit.

${ }^{12}$ Freeman note que Cohen fait de la démocratie délibérative une « conviction morale bien considérée » au sens rawlsien. Samuel Freeman, «Deliberative Democracy: a Sympathetic Comment», Philosophy and Public Affairs, vol. 29, n 4 , 2000, p. 389.

${ }^{13}$ Voir Jürgen Habermas, Faktizitât und Geltung. Beiträge zur Diskurstheorie des Rechts und des demokratischen Rechtsstaats, Francfort-sur-le-Main, Suhrkamp Verlag, 1992. Trad. par R. Rochlitz et C. Bouchindhomme : Jürgen Habermas, Droit et démocratie. Entre faits et normes, Paris, Gallimard, 1997, p. 330333. Voir aussi John Rawls, «La raison publique », op. cit., n. 2, p. 260-261 ; "L’idée de raison publique reconsidérée », n. 21, p. 168.
} 
membres s’accordent, malgré leurs différences d’opinion, pour faire de la délibération publique et libre entre citoyens égaux le mode de résolution légitime des problèmes de choix collectifs, du moins pour les questions politiques les plus importantes. Il s'agit donc d'un idéal de légitimité démocratique adapté à des sociétés pluralistes: dans un contexte de désaccords persistants, les mesures politiques légitimes sont celles qui résultent d'une délibération libre, raisonnée et égalitaire, ou qui sont requises pour qu’une telle délibération soit possible. Si le principe de légitimité, formulé à ce niveau de généralité, est commun à la plupart des théoriciens de la démocratie délibérative, ceux-ci divergent largement sur son interprétation.

En quel sens le recours à la délibération résout-il en effet le problème de la légitimité ? Il faut, pour répondre, penser la conception délibérative par contraste avec la conception agrégative de la démocratie, dans laquelle les décisions collectives sont prises en agrégeant les préférences individuelles - généralement par le vote - en l’absence de toute délibération. Cette théorie fait reposer la légitimité des décisions sur le caractère équitable de la procédure agrégative, qui accorde un poids égal aux préférences de chaque citoyen, comme l’illustre le principe « un homme, une voix ». Selon les tenants de cette conception ${ }^{14}$, en l'absence d'un consensus sur les décisions à prendre et sur les valeurs devant guider l'action collective, les citoyens ne peuvent que se mettre d'accord sur la procédure de prise de décision la mieux à même d'exprimer leur égalité et accepter comme légitimes les résultats qu’elle produit, quels qu'ils soient. Si nous sommes en désaccord profond sur l'étendue des libertés religieuses faut-il par exemple conférer aux individus le droit de choisir leurs jours fériés en fonction de leur religion ? - ou sur la protection de la liberté d'expression - faut-il par exemple interdire la pornographie ? -, il faut simplement laisser la majorité décider et accepter le résultat. La conception agrégative de la légitimité est donc strictement procédurale : la décision politique tire sa légitimité uniquement de la conformité de procédure qui l'a produite à un critère procédural, et nullement de la conformité de son contenu à un critère substantiel indépendant de la procédure ${ }^{15}$.

Les limites de cette conception apparaissent dès que l'on considère une situation où l'unanimité n’est pas spontanément produite. Quand une majorité l'emporte, pourquoi la minorité devrait-elle accepter comme légitime une décision qu’elle n’a pas voulue ? Elle n’a

\footnotetext{
${ }^{14}$ Pour un exemple de théorie agrégative qui critique la conception délibérative, voir Richard Posner, Law, Pragmatism and Democracy, Cambridge, Harvard University Press, 2003.

15 Pour une critique détaillée des théories «purement procédurales », voir Corey Brettschneider, Democratic Rights. The Substance of Self-Government, Princeton, Princeton University Press, 2007. Trad. par Y. Meinard : Corey Brettschneider, Les droits du peuple. Valeurs de la démocratie, Paris, Hermann, 2009 (à paraître).
} 
pas consenti à cette décision et n'en est pas le co-auteur. On peut certes opposer au minoritaire récalcitrant qu’il est nécessaire de prendre des décisions contraignantes pour tous, mais il répondra que cette décision particulière n’est pas nécessaire. Le caractère équitable de la règle majoritaire risque d'être une justification insuffisante dans les cas où la décision prise est manifestement injuste, dangereuse, ou attentatoire aux droits ou aux intérêts fondamentaux de certains citoyens. Le principal défaut de la conception agrégative strictement procédurale est qu'elle ne peut distinguer une telle décision des autres, car elle ne dispose d'aucun critère autre que la procédure elle-même pour évaluer la légitimité d’une décision. Elle est indifférente au contenu des préférences exprimées.

Ce n'est pas le cas de la conception délibérative de la démocratie, qui soumet la formation des volontés individuelles à l'épreuve de l'échange public de raisons. S’il y a bien agrégation des volontés individuelles à l’issue de la délibération, le vote prend ici une dimension cognitive : chaque citoyen n'affirme pas une préférence relative à son intérêt particulier - comme le font les individus de la position originelle - mais exprime son opinion sur ce qui constitue la meilleure décision pour tous. De plus, cette opinion n’est pas supposée spontanément fixée - comme dans le cas des citoyens rousseauistes qui veulent ce que veut la volonté générale - mais se forme par l’échange public de raisons. Les seules préférences pertinentes sont donc celles qui survivent à l'épreuve épistémique et morale de la délibération. Du point de vue épistémique, la délibération, en rendant publics opinions et arguments, permet à chacun de se forger une opinion informée et réfléchie : raisonnements fallacieux, positions incohérentes et présupposés erronés peuvent être éliminés. Du point de vue moral, la délibération publique, en soumettant opinions et arguments à la critique, permet à chacun de dénoncer publiquement les positions qui sacrifient de manière manifeste ses droits et ses intérêts : les options politiques incompatibles avec le statut de chaque citoyen en tant qu'il est libre et égal peuvent être éliminées. Une position affirmant par exemple que la consommation d'ouvrages pornographiques conduit automatiquement à des actes de violence sexuelle ou une position refusant seulement aux citoyens de certaines religions le droit de choisir leurs jours fériés pourraient par exemple être contestées publiquement avant que le vote n'ait lieu. La réponse délibérative au problème de la légitimité consiste donc à façonner les volontés individuelles par la délibération publique afin d'éliminer les préférences épistémiquement ou moralement contestables.

Cette réponse est toutefois paradoxale, puisque, partant du constat qu'il existe des désaccords persistants, elle réintroduit l’idéal d’unanimité comme horizon de la légitimité. En effet, la fin de la délibération, conçue comme effort collectif pour déterminer la meilleure 
option politique, est l'émergence d'un consensus moral et épistémique sur le contenu du bien commun - sur la décision qui est la meilleure pout tous. Cohen, qui souligne pourtant le caractère pluraliste de l'association démocratique, le dit clairement : la procédure délibérative idéale est « consensuelle », en ce qu'elle vise à atteindre un accord rationnellement motivé, c’est-à-dire fondé sur des raisons convaincantes pour tous les participants.

Il est vrai qu'on peut distinguer deux types distincts de recherche du consensus, inégalement problématiques. Jeremy Waldron souligne que la «logique interne de la délibération » demande que chaque participant vise la réalisation d'un consensus, car « argumenter de bonne foi revient à présenter des raisons (que l'on pense) que l'autre devrait accepter, et, pour deux personnes ou plus, persister à argumenter revient à reconnaître qu'il est possible qu'à la fin les mêmes considérations les convainquent tous ». Admettre cela ne revient pas toutefois à faire du consensus « le résultat approprié » de la délibération et à voir dans la persistance du désaccord le signe d'une incomplétude ou d'un échec du processus politique $^{16}$. Une théorie peut admettre le consensus comme une visée subjective des participants sans l'ériger en visée objective de la procédure.

Mais les théories délibératives comme celle de Cohen, même si elles reconnaissent que rien ne garantit, en théorie comme en pratique, l’unanimité des volontés, ne font pas pour autant de la recherche du consensus une simple visée subjective. Au contraire, l'efficacité même du filtrage délibératif paraît requérir l'émergence d’un consensus, car seul celui-ci peut garantir que la décision prise satisfasse effectivement les exigences épistémiques et morales sur lesquelles peuvent tomber d'accord tous les citoyens. En cas de désaccord persistant sur ce qui est épistémiquement ou moralement acceptable, comment départager, en effet, les citoyens?

Cohen ne paraît pas troublé par cette difficulté et suggère qu'en l'absence prévisible du consensus recherché, il suffit de procéder à un vote à la majorité. Mais rien ne garantit que la décision choisie finalement par la majorité possède effectivement les qualités morales et épistémiques que la délibération visait à produire. La théorie démocratique ne nous donne aucune raison de penser que la décision majoritaire corresponde systématiquement ou même tendanciellement avec la «meilleure » décision, celle qui devrait remporter l'unanimité. De nombreux théoriciens sont conscients du problème : Thomas Christiano souligne la faiblesse des interprétations épistémiques qui font de la majorité l’ « indicateur » de la bonne décision ${ }^{17}$

\footnotetext{
${ }^{16}$ Jeremy Waldron, Law and Disagreement, Oxford, Oxford University Press, 1999, p. 91-92.

17 Thomas Christiano, « The Significance of Public Deliberation », in J. Bohman et D. Regh (dir.), Deliberative Democracy. Essays on Reason and Politics, Cambridge, Mass., The MIT Press, 1997, p. 243-278.
} 
et Bernard Manin critique, à partir d'une lecture de Rousseau et Sieyès, la substitution de la règle de la majorité, simple principe pratique, à la règle d’unanimité, principe de légitimité.

C’est la supériorité affirmée de la conception délibérative sur la conception agrégative qui paraît dès lors sapée : si la délibération, n’ayant pas permis la réalisation d’un consensus, se conclut par une procédure d'agrégation, et si rien ne garantit que le résultat de cette agrégation satisfasse les exigences épistémiques ou morales portées par la délibération, en quoi la décision finalement prise se distingue-t-elle d'une décision prise en l'absence de toute délibération? En quoi est-elle plus légitime?

\section{Trois conceptions délibératives de la légitimité}

Trois réponses à cette question sont proposées par les défenseurs de la délibération. Chacune esquisse une version distincte de la démocratie délibérative, et soulève des problèmes spécifiques.

Il est tout d'abord possible de renoncer à fonder la légitimité des décisions sur les effets épistémiques et moraux de la délibération, pour la fonder plutôt sur le caractère équitable de la procédure délibérative, qui donne à chacun une chance égale de convaincre les autres participants et d'influencer la décision finale. La délibération rend plus probable la sélection d’une «bonne » décision, mais ce n'est pas la qualité de celle-ci, trop incertaine, qui la rend légitime. La théorie délibérative, comme la théorie agrégative, doit se satisfaire d'une conception strictement procédurale de la légitimité démocratique. C’est la voie suivie notamment par Christiano, qui affirme que « quels que soient les résultats des discussions, de la délibération et de la prise de décision [...] ils sont légitimes. Les résultats sont rendus légitimes parce qu'ils sont les résultats de la procédure » ${ }^{18}$. Gerald Gaus suggère de même qu'il faut renoncer à une vision consensuelle de la politique démocratique : celle-ci ne peut consister qu'en un arbitrage équitable entre points de vue irréconciliables ${ }^{19}$. Cette première solution, dessinant une version modeste de la démocratie délibérative, se heurte à la même difficulté que la théorie agrégative : les conceptions strictement procédurales de la légitimité rendent impossible la dénonciation de certaines mesures comme illégitimes du fait de leur contenu.

Une seconde réponse, exactement inverse, consiste à affirmer l'existence d'un ou de plusieurs critères substantiels indépendants qui permettent de juger le contenu des décisions

\footnotetext{
${ }^{18}$ Thomas Christiano, The Rule of the Many. Fundamental Issues in Democratic Theory, Boulder, Co, Westview Press, 1996, p. 35.

${ }^{19}$ Gerald Gaus, « Reason, Justification and Consensus : Why Democracy Can’t Have it All », in J. Bohman et D. Regh (dir.), Deliberative Democracy. Essays on Reason and Politics, op. cit., p. 205-242.
} 
politiques sur des bases morales et/ou épistémiques. De tels critères permettent d'évaluer, indépendamment de la procédure délibérative elle-même, si les décisions majoritaires menacent les droits fondamentaux des citoyens minoritaires, ou si elles reposent sur des prémisses erronées. Cette réponse a l'avantage de justifier le recours à la délibération : si celle-ci est nécessaire, c’est parce qu’elle constitue la voie la plus sûre pour s’approcher de la «bonne » décision - définie par ces critères indépendants. Les conceptions strictement procédurales ne peuvent offrir une telle justification, car si l’équité de la procédure est le seul fondement de la légitimité, pourquoi en passer par la délibération ? David Estlund souligne qu'un tirage au sort satisfait tout autant l'exigence d'équité qu'un débat public : si nous attachons de l'importance à la tenue d'un débat public contradictoire, c'est que la recherche d'un certain type de résultat et le souci de la vérité sont des dimensions essentielles de la démocratie $^{20}$. Mais cette réponse présente aussi deux inconvénients majeurs. Tout d'abord, dans un contexte de désaccords moraux et épistémiques persistants, invoquer des critères substantiels indépendants qui ne font pas eux-mêmes l'objet d'un consensus ne nous aide guère à résoudre le problème de la légitimité. Ensuite, cette version de la démocratie délibérative renonce à faire de la délibération un idéal premier : celle-ci n’est qu'un instrument pour s’approcher de ce qui fonde vraiment la décision légitime - la vérité ou la justice, par exemple.

Une dernière réponse propose une voie intermédiaire entre la conception strictement procédurale et la conception strictement substantielle de la légitimité délibérative. Elle affirme qu'il est possible de la faire dépendre aussi du contenu des décisions politiques en recourant à un critère substantiel d'évaluation qui ne soit pas pour autant indépendant de la procédure délibérative, puisqu'il s’agit de l'idéal qui la justifie ${ }^{21}$. L’idéal fondamental d'une association démocratique, que traduit la procédure délibérative, permet de juger le contenu des décisions politiques. Cohen reformule en ce sens le principe de légitimité : «les résultats sont démocratiquement légitimes si, et seulement si, ils pourraient faire l'objet d'un accord libre et raisonné entre égaux ${ }^{22}$. La " procédure délibérative idéale » qu’il élabore a certes vocation à être imitée par les institutions politiques, mais elle décrit aussi les conditions idéales sous lesquelles nous devons nous demander si une décision particulière pourrait faire l'objet d'un

\footnotetext{
${ }^{20}$ David Estlund, Democratic Authority. A Philosophical Framework, Princeton, Princeton University Press, 2007.

${ }^{21}$ Un tenant de la démocratie agrégative pourrait proposer une démarche similaire : si certains résultats sont manifestement inéquitables, ils sont incompatibles avec la valeur d’équité qui guide la procédure. Mais une telle conception n'est plus strictement agrégative au sens où nous l'entendons ici : elle fait dépendre la légitimité d'une valeur qui ne s'exprime pas uniquement dans la procédure d'agrégation.

22 Joshua Cohen, « Délibération et légitimité démocratique », p. 22 de l’édition américaine.
} 
tel accord. Ce critère, fondé sur un consensus hypothétique, doit permettre d'évaluer la légitimité des décisions en fonction de leur contenu, sans subordonner pour autant la démocratie délibérative à une autre valeur substantielle. Mais Cohen ne nous indique pas comment nous pouvons déterminer si une décision qui fait, sous des conditions non optimales, l'objet d'un désaccord, pourrait donner lieu à un consensus sous des conditions optimales. Les conceptions délibératives de Jürgen Habermas ${ }^{23}$ - dont Cohen se rapproche explicitement en s'éloignant de Rawls - et de Seyla Benhabib se heurtent au même problème. En cherchant à faire de la procédure délibérative la seule source de légitimité démocratique, et en proposant de juger les résultats des délibérations réelles à l'aune d’une procédure ou d'une situation de délibération idéale, ces théories formulent un critère de légitimité qui, malgré son attractivité théorique, se dérobe à toute application particulière. Benhabib reconnaît d'ailleurs que le théoricien de la démocratie délibérative doit faire «le pari » qu’une décision atteinte par des délibérations concrètes « sera plus rationnelle et plus légitime $»^{24}$.

Aucune de ses trois versions possibles n'est entièrement satisfaisante. C’est pourquoi de nombreuses théories paraissent osciller de l'une à l'autre. Ainsi Manin, bien qu'il dénonce l'impasse des conceptions unanimistes et qu'il insiste sur le caractère équitable de la délibération publique, qui donne à chacun sa chance de persuader les autres et accorde une place aux opinions minoritaires, cherche néanmoins à garantir la rationalité du résultat en s'appuyant sur le consensus comme visée subjective. Parce que «le processus d'argumentation se déroule face à l'auditoire universel ${ }^{25}$ des citoyens, chaque participant a selon lui intérêt à proposer des arguments acceptables par le plus grand nombre, et l’unanimité devient donc l' « horizon » de la délibération. Mais le problème est qu’il est tout aussi efficace, d'un point de vue stratégique, de former une coalition majoritaire au dépend d'une minorité que de viser un accord universel. Ici encore, rien ne garantit que la procédure avance la cause du bien commun.

\section{La raison publique et le consensus sur les raisons}

L’idée de raison publique développée par Rawls - et présentée comme un élément essentiel de la démocratie délibérative - échappe en partie à ces difficultés.

Elle spécifie le type de raisons que les citoyens d’une société bien ordonnée peuvent invoquer publiquement pour justifier les décisions politiques portant sur les questions

\footnotetext{
${ }^{23}$ Jürgen Habermas, Droit et démocratie, op. cit.

${ }^{24}$ Seyla Benhabib, « Deliberative Rationality and Models of Democratic Legitimacy », Constellations, vol. 1, 1994, p. 49.

${ }^{25}$ Bernard Manin, « Volonté générale ou délibération », op. cit., p. 89-90.
} 
constitutionnelles essentielles et sur les questions de justice fondamentale. Le devoir de civilité exige de chacun d'eux qu'il n'invoque sur ces sujets que des raisons dont il peut raisonnablement penser que les autres citoyens raisonnables - c'est-à-dire prêts à accepter des principes politiques représentant des termes équitables de coopération et prêts à leur obéir de leur plein gré à condition que les autres fassent de même - pourraient les accepter. De telles raisons sont publiques car elles reposent sur des valeurs et principes politiques qui sont communs à tous les citoyens. Les raisons privées, qui dépendent de doctrines compréhensives et sont propres à des associations particulières, telles les Églises ou les universités, sont au contraire exclues de la justification publique.

L’idée de la raison publique partage avec l'idée de la démocratie délibérative au moins quatre traits. Tout d'abord, elle constitue une conception de la légitimité : un pouvoir politique légitime est un pouvoir dont les actions sont justifiées par des raisons publiques. C’est ce qu’implique «l'idée de légitimité politique fondée sur le critère de réciprocité ${ }^{26}$. Deuxièmement, cette conception s’applique à des sociétés marquées par le fait du pluralisme raisonnable : des désaccords persistent sur la nature de la vie bonne même entre des individus raisonnables exerçant leur raison pratique sous des conditions favorables ${ }^{27}$. Troisièmement, cette conception exprime l'idéal démocratique : «dans une société démocratique, la raison publique est la raison des citoyens égaux qui, constitués en corps collectif, exercent le pouvoir politique et coercitif ultime les uns sur les autres en promulguant des lois et en amendant leur constitution $»^{28}$. Enfin, elle ne vaut que pour la justification publique : elle s'applique aux responsables politiques et aux citoyens quand ils s'engagent dans le débat public, votent ou agissent politiquement, et non aux réflexions personnelles des citoyens en tant que personnes privées. Comme les théories délibératives, la raison publique répond donc au problème de la légitimité démocratique dans les sociétés pluralistes en s’appuyant sur une forme d'argumentation publique.

A la différence de ces théories, toutefois, la raison publique ne requiert pas, pour concilier l'autonomie du citoyen et de la prise de décisions coercitives, un consensus, réel ou hypothétique, sur la décision. Car si seules des raisons publiques sont invoquées pour justifier publiquement les options politiques avancées, alors, quelle que soit l'option finalement

\footnotetext{
${ }^{26}$ John Rawls, « L’idée de raison publique reconsidérée », op. cit., p. 166.

${ }^{27}$ Rawls définit ce concept en renvoyant à un article de Joshua Cohen qui est le fruit de leurs échanges. Voir Joshua Cohen, " Moral Pluralism and Political Consensus », in D. Copp, J. Hampton, et J. Roemer (dir.), The Idea of Democracy, Cambridge, Cambridge University Press, 1993, p. 270-291.

${ }^{28}$ John Rawls, « La raison publique », op. cit., p. 261.
} 
retenue, elle sera justifiable par des raisons acceptables par tous les citoyens raisonnables, y compris par les minoritaires qui, tout en la désapprouvant, ne pourront la juger déraisonnable. Les raisons publiques sont celles que peuvent accepter des citoyens raisonnables, c'est-à-dire prêts à accepter des principes politiques représentant des termes équitables de coopération et à leur obéir de plein gré, s’ils ont l'assurance que les autres feront de même. La raison publique ne rencontre donc pas les mêmes difficultés que les théories délibératives déjà mentionnées.

Mais loin de faire l'économie de l’unanimité, elle ne fait que déplacer son lieu d'intervention, en la situant en amont du moment délibératif. Il faut en effet qu'existe un accord préalable des citoyens sur le type de raisons acceptables par tous les citoyens raisonnables, et donc sur le contenu de la raison publique. Celui-ci est donné par une conception politique de la justice, qui formule « des principes substantiels de justice pour la structure de base » de la société, c’est-à-dire un ordonnancement hiérarchisé de valeurs à partir duquel il est possible de formuler des raisons publiques, mais aussi des directives d'enquêtes, c'est-à-dire « des principes de raisonnement et des règles pour administrer la preuve à la lumière desquels les citoyens doivent décider si des principes substantiels s'appliquent correctement $»^{29}$. En l'absence d'un consensus sur une telle conception, les citoyens ne sont d'accord ni sur les valeurs ni sur les types de raisonnement qui sont acceptables dans la justification publique. C'est pourquoi il importe que les valeurs et les directives d'enquête d'une conception politique forment un ensemble «complet »: les premières doivent pouvoir être équilibrées, combinées, voire unifiées, de sorte à pouvoir déterminer au moins une réponse raisonnable à toute question constitutionnelle essentielle ou à toute question de justice fondamentale. La limitation de la raison publique à ces seules questions paraît d'ailleurs motivée par le même souci. La raison publique ne peut s'appliquer à toutes les questions de la politique ordinaire, car elle suppose l'existence d'un consensus assez large sur son contenu pour que celui-ci fournisse une ou plusieurs réponses raisonnables ${ }^{30}$.

\section{La délibération subordonnée à l'équité}

En substituant à la recherche d'un consensus sur la décision par la délibération le présupposé d’un consensus sur les raisons préalable à la délibération, la raison publique

\footnotetext{
${ }^{29}$ Ibid., p. 272.

30 A défaut d'être toujours conclusive, la raison publique doit donc au moins être déterminée. Sur cette distinction, voir Gerald Gaus, Justificatory Liberalism. An Essay on Epistemology and Liberal Theory, New York, Oxford University Press, 1996.
} 
procède en réalité comme la politique démocratique de la Théorie de la justice: elle subordonne la délibération à l'équité. L'idéal d’une association de citoyens s’autogouvernant par l'échange public de raisons n'est pas ici premier, mais dérive de l'idéal plus fondamental d'un système équitable de coopération - et de l'exigence de réciprocité qui lui est associée. Pour que la raison d’une société puisse être véritablement publique, il faut que cette société soit déjà une société bien ordonnée, organisée en fonction d'une conception politique, telle la théorie de la justice comme équité, qui soit partagée par tous ses membres. Rawls indique d'ailleurs que son but est d'offrir « une formulation acceptable » de l'idée de la raison publique « en montrant comment elle fait partie d'une conception politique de la justice libérale au sens large $»^{31}$.

Certes, il rattache aussi cette idée directement à l'idée d'une association démocratique, et l'on pourrait chercher à minorer l'écart entre démocratie délibérative raison publique, en opposant trois objections à la lecture selon laquelle cette dernière subordonne la délibération à l’équité. Mais aucune de ces objections n’est convaincante.

Selon une première objection, la raison publique ne peut pas supposer un consensus préalable sur une conception politique de la justice, car elle a pour effet de faciliter un tel consensus. Rawls insiste sur le caractère stabilisateur de l'exercice de la raison publique, qui consolide le consensus par recoupement et encourage le développement du sens de la justice des citoyens : « la conception politique de la justice et son idéal de la raison publique se renforcent mutuellement $»^{32}$. Mais ce renforcement ne remet pas en cause la primauté de la conception sur son idéal : certes l'exercice de la raison publique affermit l'adhésion aux valeurs et aux règles d’enquête publiques, mais celui-ci suppose déjà celles-là.

Selon une seconde objection, la raison publique ne suppose un tel consensus que dans le cas idéal d'une société déjà bien ordonnée, et non dans le cas non idéal d'une société qui ne l'est pas encore. Dans ce dernier cas, c'est la version inclusive de la raison publique, qui autorise les citoyens à invoquer des raisons non publiques quand cela renforce l'idéal de la raison publique, qui s’applique. L'usage public d'arguments religieux par Martin Luther King ou par les abolitionnistes américains est ainsi justifiable, car il a renforcé la raison publique sur le long terme, en contribuant à «faire naître une société juste et bien ordonnée dans laquelle l'idéal de la raison publique pourrait enfin être respecté » ${ }^{33}$. Mais le fait qu'une telle justification soit possible a posteriori ne fait que confirmer la primauté logique de la

\footnotetext{
${ }^{31}$ John Rawls, « La raison publique », op. cit., p. 260.

${ }^{32}$ Ibid., p. 303.

${ }^{33}$ Ibid., p. 298.
} 
conception politique de la justice : c’est seulement sur la base de la conception politique qui s’est finalement imposée, permettant que l'idéal de la raison public soit " enfin respecté », que cette justification est possible.

Selon une dernière objection, la raison publique ne suppose pas un consensus sur une conception politique, car Rawls, en reconsidérant sa théorie, précise que le contenu de la raison publique est donné par une famille de conceptions politiques libérales de la justice. Cette objection est difficile à éprouver, car il n'élabore guère les conditions sous lesquelles différentes conceptions de la justice peuvent respecter les règles de la raison publique. Charles Larmore suggère la lecture suivante ${ }^{34}$ : les citoyens justifient publiquement leurs actes et décisions politiques quand les raisons formulées ne dépendent que du contenu commun aux différentes conceptions politiques de la justice apparentées. En d’autres termes, un consensus sur l'intégralité d'une conception politique n’est pas nécessaire, il suffit d'un consensus sur les valeurs et les directives d'enquête qui sont communes à différentes conceptions. Mais cet aménagement de la théorie ne fait que substituer à un consensus un autre consensus, tout aussi exigeant si la condition de complétude est maintenue, et auquel est toujours subordonnée la délibération.

Celle-ci, loin d’être libre, est donc contrainte par des principes indépendants de l’idéal délibératifs. Ceci ne constitue pas un problème en soi pour la théorie rawlsienne, mais signifie que la raison publique, quoiqu'elle accorde une place à la délibération, n’est pas une conception de la démocratie délibérative. Elle ne vise ni l'émergence d'un consensus effectif ni l'approximation d'un consensus hypothétique sur la décision à prendre, mais repose sur un consensus préalable portant sur le type de raisons acceptables par tous. Elle ne doit pas opérer une transformation des préférences individuelles, mais plutôt une sélection du type d’arguments invoqués dans le raisonnement public. Elle ne fait pas de la délibération la source fondamentale de la légitimité, mais adosse cette dernière à une conception indépendante exprimant l'idéal d’équité. Bref, tandis que la démocratie délibérative lie la légitimité à un processus dynamique d'échanges de raisons entre citoyens dont le but est de transformer les volontés individuelles afin de s'approcher d'un accord normatif, la raison publique est un principe régulateur qui impose des limites à la manière dont les citoyens devraient raisonner sur certaines questions publiques en fonction d'une conception politique supposée commune. Il n'est donc pas étonnant que Rawls présente les raisonnements de la Cour Suprême comme l'exemple paradigmatique d'exercice de la raison publique : l'échange

\footnotetext{
${ }^{34}$ Charles Larmore, « Public Reason », in S. Freeman (dir.), The Cambridge Companion to Rawls, Cambridge, Cambridge University Press, 2003, p. 389.
} 
public d'arguments entre citoyens n'est qu'une modalité parmi d'autres de son exercice et n'est pas même la plus importante ${ }^{35}$.

\section{Deux conceptions inconciliables de la légitimité}

Le constat de ces divergences majeures entre démocratie délibérative et raison publique peut, au-delà de la seule exégèse rawlsienne, donner lieu à deux conclusions opposées : soit ces deux idées renvoient à deux conceptions inconciliables de la légitimité démocratique, soit elles constituent deux variantes d'une même conception de la légitimité qui devraient pouvoir être articulées, au prix de quelques aménagements, au sein d'une théorie cohérente de la démocratie.

Les tenants de la première position nient que la raison publique soit une conception authentiquement «délibérative » et l'érigent même en contre-modèle. Cooke et Benhabib soulignent que la raison publique est un principe de contrainte s'exerçant sur les raisonnements individuels plutôt qu'une forme de raisonnement collectif. Cooke reproche par ailleurs à Rawls d'adopter une vision excessivement statique des volontés individuelles et des désaccords substantiels, que révèle la volonté d'exclure entièrement du débat public les conflits moraux supposés insolubles ${ }^{36}$. Benhabib, de son côté, accuse Rawls de trop restreindre la délibération, en limitant non seulement les raisons invocables, mais aussi les questions discutées et les lieux de la discussion - la raison publique ne s’applique pas à la société civile et aux associations. Toutes deux rejettent la perspective rawlsienne, pour mieux se rapprocher de la théorie de Habermas, qui conçoit la délibération que comme un processus non contraint d'autodétermination ${ }^{37}$. Mais en refusant de subordonner l'idéal délibératif, ces théories rencontrent le problème identifié à la section 3 : elles ne disposent, pour évaluer le contenu des décisions prises, que de l’idéal délibératif lui-même.

\footnotetext{
${ }^{35}$ La seconde formulation de la raison publique limite d'ailleurs son exercice à trois cas : les raisonnements des juges, les justifications des responsables gouvernementaux, et les discours des candidats et des responsables de partis. Les citoyens intervenant publiquement ne sont plus mentionnés.

${ }^{36} \mathrm{Il}$ n'est d'ailleurs pas certain que la stratégie d'évitement que constitue la raison publique soit le meilleur moyen pour les citoyens de se témoigner mutuellement du respect, comme le suggère Rawls. Considérer autrui comme étant capable de réviser sa conception du bien et accepter en conséquence de débattre avec lui de ces questions est aussi une manière de le traiter avec respect. Voir Speranta Dumitru, «La raison publique : une conception politique et non épistémologique ? », Archives de philosophie du droit, vol. 49, 2005, p. 233-244.

37 Jürgen Habermas, « Volkssouveränität als Verfahren. Ein normativer Begriff von Öffentlichkeit », Merkur, 1989. Trad. par M. Hunyadi : Jürgen Habermas, «La souveraineté populaire comme procédure. Un concept normatif d'espace public », in C. Girard et A. Le Goff, La démocratie délibérative. Textes fondamentaux, op. cit. Habermas reproche à la politique rawlsienne de contraindre la délibération par des principes préalables déterminés de manière monologique par le philosophe politique : Jürgen Habermas et John Rawls, Débat sur la justice politique, trad. C. Audard et R. Rochlitz, Paris, Cerf, 1997.
} 
Les partisans de la seconde position se proposent au contraire d'incorporer certains éléments de la raison publique à leur théorie délibérative, mais le succès de ces tentatives de conciliation est douteux. Ainsi James Bohman revendique-t-il autant l'influence de Rawls que celle de Habermas, quand il propose de reformuler les problèmes de la théorie normative à partir d'une caractérisation pragmatique des faits sociaux (tel le fait du pluralisme raisonnable). Mais si Bohman mobilise l’idée du raisonnable, il juge que la profondeur des désaccords moraux est telle que le champ de ce qu'il est « raisonnable pour tous d'accepter » est trop étroit pour justifier des décisions politiques. Le contenu épistémique et moral de la raison publique ne pouvant que faire l'objet de désaccords, il faut accepter une « pluralité des raisons publiques ${ }^{38}$. Amy Gutmann et Dennis Thompson proposent également, en s'inspirant largement de Rawls, d'affronter les conflits moraux persistants en mettant en œuvre un processus public de délibération normé par l'idéal d'équité mais aussi par des principes politiques indépendants. Mais ils refusent d'exclure ces principes du champ de la délibération : bien qu'indispensables, ceux-ci doivent pouvoir être révisés. Les résultats de la délibération publique sont donc toujours provisoires et peuvent redéfinir les limites de ce qui vaut comme une raison publique ou une position raisonnable ${ }^{39}$. Tentant d'incorporer la raison publique à la démocratie délibérative sans contraindre pour autant la délibération, Gutmann et Thompson, comme Bohman, sont amenés à redéfinir le sens du raisonnable et à renoncer au fondement même de l’idée rawlsienne. La délibération, si elle est la source première de la légitimité politique, ne peut pas présupposer une définition du raisonnable qui soit fixée indépendamment d'elle.

Les obstacles rencontrés par ses tentatives d'incorporation ne sont pas fortuites. Il est simplement impossible de concilier d'une part, le refus de restreindre la procédure délibérative par des principes indépendants de l'idéal délibératif - refus qui est motivé par une interprétation de la démocratie comme idéal d'autogouvernement par l'échange public de raisons - et d'autre part la limitation des raisons invocables au cours de cette procédure par des principes indépendants de justice - limitation qui est imposée par une interprétation de la

\footnotetext{
38 James Bohman, Public Deliberation. Pluralism, Complexity and Democracy, Cambridge, The MIT Press, 1996. Sur le lien entre faits sociaux et théorie normative voir James Bohman, «Realizing Deliberative Democracy as a Mode of Inquiry: Pragmatism, Social Facts and Normative Theory Journal of Speculative Philosophy », n 18, vol. I, 2004, p. 23-43. Trad. par C. Girard : James Bohman, "Réaliser la démocratie délibérative comme mode d'enquête: pragmatisme, faits sociaux et théorie normative », Tracés, vol.15, http://traces.revues.org/index883.html.

${ }^{39}$ Amy Gutmann et Dennis Thompson, Democracy and Disagreement, Cambridge, Harvard University Press, 1996 ; «Why is Deliberative Democracy Different ? », Social Philosophy and Policy, 2000, vol. 17, n¹, p. 161180. Trad. par L. Gagné : «Pourquoi la démocratie délibérative est-elle différente ? », Philosophique, vol. 29, n², 2002, http://www.erudit.org/revue/philoso/2002/v29/n2/006250ar.html.
} 
démocratie comme système de coopération équitable. Ces deux conceptions de la légitimité démocratique sont manifestement inconciliables.

Cela apparaît très clairement dans la reformulation que propose Joshua Cohen de sa théorie délibérative, laquelle constitue la tentative la plus radicale de conciliation de ces deux conceptions. Il propose toujours de fonder la légitimité des décisions à la fois sur la procédure délibérative qui les produit et sur la conformité de leurs contenus à l’idéal guidant cette procédure. Toutefois, le critère de légitimité n’est plus la capacité des décisions à faire l’objet d’un accord rationnel sous des conditions idéales. Dans la procédure délibérative idéale, les citoyens doivent désormais « défendre et critiquer les institutions et les programmes par des considérations que les autres ont des raisons d'accepter, étant donné le fait du pluralisme raisonnable et la supposition que les autres sont raisonnables ${ }^{40}$. Cette reprise terme à terme de l'idée de la raison publique sous le nom de «principe d’inclusion délibérative » permet à Cohen de justifier le recours à la règle de la majorité en cas de désaccord persistant, alors que la formulation antérieure de sa théorie ne le permettait pas - comme nous l'avons vu à la section 2. En effet, si toutes les options avancées le sont sur la base de raisons acceptables par tous, le fait qu'une option particulière soit soutenue par la majorité peut constituer une raison suffisante pour tous les citoyens - y compris les minoritaires - de la juger légitime. Mais ce critère d'acceptabilité des raisons est tout aussi problématique que l'ancien critère d’acceptabilité de la décision : comment déterminer si les raisons avancées sont acceptables par tous, quand elles ne sont pas acceptées par tous $?^{41}$

Cohen, comme Rawls, conçoit une raison acceptable comme une raison qui est telle qu'un citoyen raisonnable peut penser que les autres citoyens raisonnables l'accepteront. Mais alors que Rawls définissait le raisonnable par la compatibilité avec l'engagement à participer à un système équitable de coopération, et pouvait donc charger une conception politique de la justice d'en préciser les limites, Cohen ne peut que le définir par la compatibilité avec l'engagement à participer à une association s’autogouvernant par la délibération publique, car il refuse toujours de subordonner la délibération à l'équité. Il dispose bien d'un critère substantiel pour évaluer le contenu des raisons et des décisions avancées, mais celui-ci est bien moins déterminé que ne l'est le critère rawlsien : là où la conception politique rawlsienne

\footnotetext{
${ }^{40}$ Joshua Cohen, « Procedure and Substance in Deliberative Democracy », in S. Benhabib (dir.), Democracy and Difference: Changing Boundaries of the Political, Princeton, Princeton University Press, 1996, p. 100. Rawls renvoie, dans «L'idée de la raison publique reconsidéré », à une version augmentée de cet article : Joshua Cohen, « Democracy and Liberty », in J. Elster (dir.), Deliberative Democracy, Cambridge, Cambridge University Press, 1998, p. 185-223.

${ }^{41}$ La question pourrait d'ailleurs aussi être posée quand les raisons sont acceptées par tous : l'acception effective ne suffit pas à prouver l'acceptabilité. Le critère de légitimité n’est pas n'importe quel accord, mais un accord raisonnable.
} 
précise le sens des valeurs qu'elle contient, offre des moyens de les hiérarchiser, et propose des règles épistémiques pour les mobiliser dans des raisonnements de justification, la conception de Cohen n’offre qu’un idéal général, exprimé par une procédure idéale ${ }^{42}$.

Cet idéal suffit certes à rejeter certaines raisons et décisions. Ainsi, des raisons qui invoqueraient une inégalité de droits entre citoyens - selon des distinctions raciales, par exemple - ou qui justifieraient un traitement inégal des groupes - en légalisant par exemple l'esclavage - sont manifestement déraisonnables au sens de Cohen : elles ne sont pas compatibles avec l'idée d'une association de citoyens libres et égaux se gouvernant par la délibération. Si des débats réels toléraient de telles raisons ou entérinaient de tels résultats, ils pourraient être dénoncés comme illégitimes du seul fait de leur contenu.

Mais ce résultat est trop limité, et il n’est pas certain que l’idéal délibératif soit assez déterminé pour protéger de la sorte tous les droits et intérêts fondamentaux que nous souhaitons voir préserver des errements des délibérations effectives. Cohen affirme qu'il l'est assez pour protéger par exemple largement la liberté religieuse et la liberté d'expression. Parce que les obligations religieuses constituent pour certains citoyens raisonnables des contraintes rigoureuses auxquelles ils peuvent difficilement se dérober, ils ne peuvent accepter qu'on les prive des libertés leur permettant d'honorer ces obligations, à moins que ne soit avancée une raison au moins aussi impérieuse que l'obligation religieuse. Il faudrait par exemple une raison véritablement impérieuse pour justifier qu’on les prive du droit de prendre leurs jours de congé lors des fêtes religieuses. De même, parce qu'un artiste peut éprouver comme un besoin fondamental le désir d'explorer dans ses œuvres, de la manière la plus crue, la dimension sexuelle de la vie humaine, il faudrait avancer une raison véritablement impérieuse pour interdire la pornographie. Seules des raisons de ce type peuvent être acceptables par tous quand il s’agit de réduire la liberté d'expression, car celle-ci protège des intérêts essentiels pour les individus. Des politiques qui restreindraient ces libertés sur la base de raisons qui ne sont pas impérieuses ne traiteraient pas les individus concernés comme des citoyens égaux aux autres et libres de faire valoir leurs droits et leurs intérêts fondamentaux dans la délibération publique.

Cette mise en œuvre du principe d'inclusion délibérative décrit certes de manière plus détaillée que ne le faisait la première formulation de l'idéal délibératif la manière dont celui-ci permet d'évaluer le contenu de décisions politiques particulières. Mais comment déterminer

\footnotetext{
${ }^{42}$ Sur ce point, voir Samuel Freeman, «Deliberative Democracy: a Sympathetic Comment », Philosophy and Public Affairs, vol. 29, n 4, 2000, p. 389-396 ; Bertrand Guillarme, Rawls et l'égalité démocratique, Paris, PUF, 1995, p. 214.
} 
quelles obligations sont rigoureuses, quels intérêts sont fondamentaux, quelles raisons sont impérieuses ? Et comment les mesurer entre eux pour déterminer lequel l'emporte sur l'autre, aux yeux d'un citoyen raisonnable ? En l'absence d'un idéal indépendant et consensuel supportant cette détermination, il semble que cette tâche incombe aux participants à la délibération. Nous nous heurtons une nouvelle fois à la même difficulté : sur quelles bases trancher les désaccords effectifs entre citoyens à ce sujet $?^{43}$

Mais surtout, cette indétermination du raisonnable remet en cause la capacité même de la procédure délibérative à être source de légitimité. On comprend pourquoi les citoyens minoritaires rawlsiens peuvent reconnaître comme légitime une décision qu’ils n’ont pas choisie mais qui est justifiée d'une manière acceptable par eux. Il est plus difficile de comprendre pourquoi les citoyens minoritaires de Cohen devraient reconnaître la décision majoritaire comme légitime si, tout en partageant les valeurs politiques invoquées par sa justification, ils jugent cette justification erronée - parce qu'ils ne sont pas d'accord sur l’interprétation, la hiérarchisation ou le mode de mobilisation de ces valeurs.

Dès lors le détour par le raisonnable, qui consiste à substituer un consensus sur les raisons acceptables à un consensus hypothétique sur la décision, est inutile et ne modifie en rien la logique de son modèle délibératif : il faut bien supposer que la délibération produit le consensus qu'elle ne peut présupposer. En intégrant l’idée de la raison publique à la théorie délibérative, il ne peut qu’annuler le contenu de la première s’il veut préserver celui de la seconde.

\section{Conclusion}

La démocratie délibérative et la raison publique constituent deux conceptions inconciliables de la légitimité démocratique. Tandis que la première comprend d'abord la démocratie comme un idéal d'autogouvernement par la délibération publique, la seconde la conçoit avant tout comme un idéal de coopération équitable. Tandis que la première refuse de subordonner la délibération à des principes indépendants et en fait la source fondamentale de toute légitimité, la seconde restreint la délibération en en excluant les raisons qui sont incompatibles avec une norme du raisonnable définie de manière indépendante par une conception politique de la justice. En conséquence, là où la première vise un consensus épistémique et moral sur la décision à prendre et compte sur la transformation des volontés individuelles pour le faire émerger, la seconde présuppose un consensus préalable sur le type

\footnotetext{
${ }^{43}$ Notons que la raison publique rawlsienne ne nous aide pas plus à répondre à ces questions, si on considère qu'elles ne constituent pas des questions constitutionnelles essentielles ni des questions de justice fondamentale.
} 
de raisons acceptables dans le forum public, et compte sur le sens de la justice des citoyens pour ne pas introduire dans celui-ci des raisons inacceptables. La limite de la première tient à ce qu'elle court le risque du déraisonnable : elle ne peut garantir que la délibération publique protège, même sous des conditions idéales, tous les droits et les intérêts individuels que nous souhaitons voir protéger. La limite de la seconde tient au contraire à ce que, en imposant des garde-fous à la délibération afin de prévenir de telles dérives, elle s’interdit de justifier ceux-ci au regard de l'idéal délibératif d’autogouvernement. Une théorie de la démocratie ne peut concilier ces deux conceptions de la légitimité - il lui faut choisir.

Il serait erroné de voir dans l'écart séparant la démocratie de la raison publique, l'opposition d'une théorie utopique, ou idéale, à une théorie réaliste, ou non idéale (quelle que soit d'ailleurs la répartition des rôles que l'on ait en tête). Ces deux conceptions se situent au niveau le plus fondamental de la théorie politique normative, celui de l'élucidation des idéaux normatifs, et supposent toutes deux des citoyens sincères et désireux de coopérer, parmi bien d'autres choses. Mais surtout, l'exigence que la délibération produise un consensus raisonné ou s'en approche, n'est ni plus ni moins forte que l'exigence qu'elle puisse s'appuyer sur une conception politique de la justice partagée par tous. Si le lieu de l'accord unanime est dans chaque cas différent, ces deux hypothèses sont également coûteuses en termes de plausibilité. Elles posent sans doute des difficultés différentes au philosophe politique qui ne se contente pas de décrire une situation idéale, mais se propose de montrer comment nous pourrions tenter d'atteindre celle-ci depuis la situation présente. Cette route, malheureusement, est encore trop rarement suivie par les théories de la démocratie.

Il serait tout aussi erroné de voir dans l'écart entre ces deux conceptions l'opposition d'un modèle constitutionnel à un modèle non constitutionnel. La théorie délibérative se satisfait tout autant d'une Constitution que la raison publique, quoiqu'elle ne lui donne pas le même sens. La Constitution d'une démocratie délibérative affirme bien des principes politiques supposés commun à tous les citoyens, mais ces principes ne tirent leur justification que du fait qu'ils ont été produits par le processus délibératif ou qu’ils sont requis pour que celui-ci soit possible. Ils seront donc sans doute plus aisément révisables - ce qui signifie également plus vulnérables aux caprices de la majorité, qu’elle soit simple ou qualifiée - que les principes constitutionnels définis indépendamment du processus délibératif. Mais ils seront aussi plus justifiés au regard de l’idéal d'autogouvernement.

Ce qui importe, du point de vue de la théorie délibérative, est de ne pas confondre la délibération publique, qui est le moment d'un effort réciproque de persuasion en vue de l'autodétermination collective, et la vérification de la justifiabilité publique, qui est le moment 
du contrôle de la compatibilité de la décision produite avec les principes politiques reconnus par les délibérations antérieures comme étant ceux de l'association politique. La délibération ne peut pas faire ce dernier travail - il incombe plutôt à une cour constitutionnelle vérifiant que la décision majoritaire qui a conclu le débat public est compatible avec les principes de la Constitution, c’est-à-dire avec les plus importantes interprétations de l’idéal démocratique que les citoyens aient jusqu'ici produites en délibérant. La démocratie délibérative peut, en d'autres termes, intégrer une procédure de vérification de la justifiabilité publique des décisions qui soit distincte de la délibération publique sans être pour autant indépendante d'elle. Elle a d'autant moins besoin de fixer à l'avance les frontières définitives de l'accord et du désaccord, de l'acceptable et de l'inacceptable. Elle peut laisser sa chance à la persuasion $^{44}$.

\footnotetext{
${ }^{44}$ Ce travail a été réalisé avec le soutien du programme DELICOM de l’ANR (JC-JC 05). Il a grandement bénéficié des commentaires de Jean-Fabien Spitz, Speranta Dumitru, Christopher Hamel et Florence Hulak sur une première version de ce texte, ainsi que de discussions avec Bernard Manin, Philippe Urfalino et Francesco Callegaro lors du séminaire « Institution de la délibération » à l’EHESS.
} 